\title{
Ferroptosis in p53-dependent oncosuppression and organismal homeostasis
}

\author{
L Galluzzi ${ }^{1,2,3,4,5,8}$, JM Bravo-San Pedro ${ }^{1,2,3,4,5}$ and G Kroemer ${ }^{\star, 1,2,3,4,6,7,7}$
}

Cell Death and Differentiation (2015) 22, 1237-1238; doi:10.1038/cdd.2015.54

Tumor protein p53 (TP53, hereafter referred to as p53) is an oncosuppressive transcription factor that mediates critical homeostatic functions. For a long time, the oncosuppressive activity of p53 was attributed to its capacity to initiate cell cycle arrest (be it temporary or permanent), and/or regulated cell death $(R C D)$ in response to stress, via transcriptional and transcription-independent mechanisms. Thus, p53 was thought to operate in favor of organismal homeostasis by keeping under check or eliminating potentially dangerous cells. ${ }^{1}$ More recent data have challenged this notion by suggesting that the oncosuppressive functions of p53 mainly originate from its ability to regulate metabolism in baseline conditions. ${ }^{2-4}$ Now, investigators from the Columbia University (New York, NY, USA) identified ferroptosis, an iron-dependent form of necrotic RCD, as an additional mechanism through which stress-activated p53 may maintain organismal homeostasis. $^{5}$

Using a cell line stably transduced with a tetracyclineinducible p53-encoding construct, Jiang et al. identified solute carrier family 7 (cationic amino-acid transporter, $\gamma+$ system), member 11 (SLC7A11), as a novel transcriptional target of p53. Accordingly, the promoter of SLC7A11 contains a p53responsive element, and p53 activation by tetracycline resulted in the time-dependent repression of SLC7A11, along with the expression of other well-known p53-regulated genes. ${ }^{5}$ This effect was found to directly rely on p53, as it could not be appreciated in the presence of a p53-specific short-hairpin RNA. However, it did not relate to the ability of p53 to transactivate cell cycle-arresting and RCD-inducing genes, as it was retained by a $\mathrm{p} 53$ mutant $\left(\mathrm{p} 53^{3 \mathrm{KR}}\right)$ that is deficient for these functions (as it bears $R \rightarrow K$ substitutions in three lysine residues that are critical for the post-translational regulation of p53 by acetylation). ${ }^{5,6}$ SLC7A11 is a component of the $X_{C}^{-}$ system, a plasma membrane multiprotein transporter that mediates the $\mathrm{Na}^{+}$-independent uptake of extracellular cystine in exchange of cytosolic glutamate. ${ }^{7}$ In line with this notion, p53 ${ }^{3 K R}$ expression caused a reduction in intracellular cystine concentrations, whereas the homologous knockout of Trp53 resulted in a $60 \%$ increase in baseline cystine levels. ${ }^{5}$ Importantly, inhibition of the $\mathrm{X}_{\mathrm{C}}^{-}$system has previously been mechanistically linked to the ability of one peculiar molecule, namely, erastin, to cause ferroptosis in cancer cells expressing mutant RAS. ${ }^{8,9}$

At odds with their Trp53-1- counterparts, wild-type (WT) and $\mathrm{p} 53^{3 \mathrm{KR}}$-expressing mouse embryonic fibroblasts (MEFs) were found to be sensitive to cell death induction by erastin. ${ }^{5}$ Importantly, such a lethal response was completely prevented by the ferroptosis inhibitor ferrostatin-1 (Fer-1), but it was not influenced by the wide-spectrum caspase inhibitor Z-VAD$\mathrm{fmk},{ }^{10,11}$ the autophagy inhibitor 3-methyladenine ${ }^{11,12}$ or the necroptosis inhibitor necrostatin-1. ${ }^{13,14}$ Additional inhibitors of ferroptosis including deferoxamine, $N$-acetyl-cysteine, $\beta$-mercaptoethanol and 1,4-diamino-2,3-dicyano-1,4-bis[2-aminophenylthio]butadiene (U0126) all inhibited erastin-induced $\mathrm{RCD}$ in $\mathrm{p} 53^{3 \mathrm{KR}}$-expressing MEFs. Moreover, the transgeneenforced overexpression of SLC7A11 quenched the loss of colony-forming capacity exhibited by $\mathrm{p} 53^{3 \mathrm{KR}}$-expressing human cancer cells (in the absence of overt RCD). These findings suggest that ferroptosis can ensue the p53dependent transcriptional repression of SLC7A11 in response to stress. However, the authors did not test whether cyclosporine $A$, an inhibitor of mitochondrial permeability transition (MPT)-dependent regulated necrosis (a major, physiologically relevant form of nonapoptotic RCD), or the absence of Ppif (coding for the key MPT regulator cyclophilin D), ${ }^{15}$ would influence the ability of erastin to kill p53- or p53 ${ }^{3 K R}$-expressing cells. As chemical inhibitors of ferroptosis (as well as cystine) share a robust antioxidant activity, which precise RCD modality is activated in this context remains unclear (although previous data support the notion that MPT-driven regulated necrosis and ferroptosis are mechanistically distinct). ${ }^{7}$

A consistent proportion of human neoplasms exhibit increased SCL7A11 levels, irrespective of TP53 mutational status, suggesting that SCL7A11 expression may support oncogenesis. In support of this hypothesis, the oncosuppressive functions of $\mathrm{p} 53^{3 \mathrm{KR}}$ in xenograft tumor models were significantly impaired upon SLC7A11 re-expression. Moreover, the lethal phenotype associated with the homologous

\footnotetext{
${ }^{1}$ Equipe 11 labellisée Ligue contre le Cancer, Centre de Recherche des Cordeliers, 75006 Paris, France; ${ }^{2}$ INSERM, U1138, Centre de recherche les Cordeliers, 75006 Paris, France; ${ }^{3}$ Université Paris Descartes/Paris V, Sorbonne Paris Cité, 75006 Paris, France; ${ }^{4}$ Université Pierre et Marie Curie/Paris VI, 75006 Paris, France; ${ }^{5}$ Gustave Roussy Comprehensive Cancer Institute, 94805 Villejuif, France; ${ }^{6}$ Metabolomics and Cell Biology Platforms, Gustave Roussy Comprehensive Cancer Institute, 94805 Villejuif, France and ${ }^{7}$ Pôle de Biologie, Hôpital Européen Georges Pompidou, AP-HP, 75015 Paris, France

*Corresponding author: G Kroemer, INSERM, U1138, Centre de Recherche les Cordeliers, 15 rue de l'Ecole de Medicine, F-75006 Paris, France. Tel: +33 1442776 67; Fax: +33 1442776 74; E-mail: kroemer@orange.fr

${ }^{8}$ These authors contributed equally to this work.
} 
knockout of transformed mouse 3T3 cell double minute 2 (Mdm2, coding for a key p53 inhibitor) appeared to rely, at least in part, on the capacity of p53 to stimulate ferroptosis. Indeed, the intraperitoneal administration of Fer-1 to pregnant mice partially relieved the lethal developmental defects associated with the $\operatorname{Trp} 53^{3 K R / 3 K R} M d m 2^{-1-}$ genotype. ${ }^{5}$ This suggests that the activation of p53 induced by the absence of $M d m 2$ may be embryonically lethal owing to the activation of ferroptosis. Jiang et al., however, did not test whether their observations also apply to $M d m 2^{-1-}$ mice expressing WT p53. Thus, the suggested link between the $\mathrm{Mdm2}^{-1}$ genotype and ferroptosis-mediated embryonic lethality remains to be formally demonstrated.

Given the implication of ferroptosis in oxidative stress responses, Jiang et al. tested the sensitivity of $p 53^{3 \mathrm{KR}}$ expressing cells to RCD induction by tert-butyl hydroperoxide $(\mathrm{TBH}) .^{5}$ In the presence of $\mathrm{p} 53^{3 \mathrm{KR}}$, cancer cells were hypersensitive to $\mathrm{TBH}$, a lethal response that could be blocked by Fer-1, but not by Z-VAD-fmk, 3-methyladenine or necrostatin-1. Again, the authors did not include cyclosporine $A$ in their experimental determinations. Sublethal TBH concentrations strongly synergized with the p53-activating molecule nutlin-3 in killing human osteosarcoma U2OS cells (which express WT p53), and this synergistic interaction could be completely blocked by Fer-2 and deferoxamine. Finally, the cytotoxic activity of $\mathrm{TBH}$ and erastin was significantly reduced in MEFs obtained from BAC transgenic mice that express increased amounts of SLC7A11. Taken together, these data point to the existence of a p53 $\rightarrow$ SLC7A11 signaling axis that mediates lethal effects in the context of failing antioxidant defenses.

The molecular cascade highlighted by Jiang et $a l^{5}$ may contribute to the oncosuppressive functions of p53. However, there are at least two major questions that await urgent clarification. First, does Fer-1 inhibit embryonic degeneration

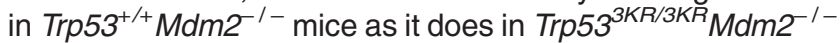
animals? Second, what is the contribution of CYPDdependent MPT-driven regulated necrosis in this setting? Irrespective of these incognita, the findings discussed above lend fresh support to the notion that the oncosuppressive activity of p53 involves the elimination of potentially oncogenic cells by senescence or RCD (depending on the experimental model). This said, a large body of evidence indicates that p53 mediates oncosuppressive functions also by avoiding, rather than responding to, the potentially oncogenic degeneration of cellular functions. ${ }^{2-4}$ Most likely, the robust tumor-suppressive activity of p53 reflects its unique ability to preserve both cellular and organismal homeostasis (Figure 1).

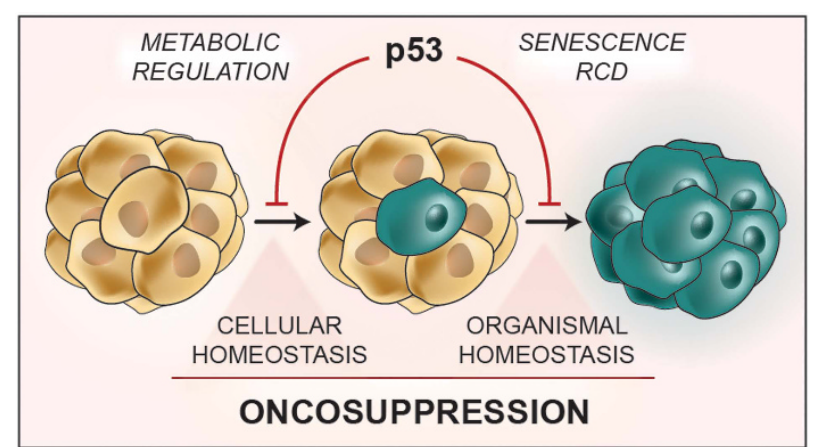

Figure 1 Oncosuppressive functions of p53. For a long time, the oncosuppressive activity of p53 was mainly ascribed to its capacity to keep under check or eliminate damaged, and hence potentially oncogenic, cells. Thus, p53 was viewed as a guardian of organismal homeostasis that operates at the expenses of individual cells. More recently, it has become clear that p53 mediates prominent oncosuppressive effects also by preventing (rather than simply responding to) the potentially tumorigenic degeneration of cellular functions. Thus, p53 appears to suppress tumorigenesis by preserving both cellular and organismal homeostasis. $\mathrm{RCD}$, regulated cell death

\section{Conflict of Interest}

The authors declare no conflict of interest.

Acknowledgements. We are supported by the Ligue contre le Cancer (équipe labelisée); Agence National de la Recherche (ANR)-Projets blancs; ANR under the frame of E-Rare-2, the ERA-Net for Research on Rare Diseases; Association pour la recherche sur le cancer (ARC); Cancéropôle lle-de-France; Institut National du Cancer (INCa); Fondation Bettencourt-Schueller; Fondation de France; Fondation pour la Recherche Médicale (FRM); the European Commission (ArtForce); the European Research Council (ERC); the LabEx Immuno-Oncology; the SIRIC Stratified Oncology Cell DNA Repair and Tumor Immune Elimination (SOCRATE); the SIRIC Cancer Research and Personalized Medicine (CARPEM); and the Paris Alliance of Cancer Research Institutes (PACRI).

1. Khoo KH, Verma CS, Lane DP. Nat Rev Drug Discov 2014; 13: 217-236.

2. Berkers CR et al. Cell Metab 2013; 18: 617-633.

3. Green DR, Galluzzi L, Kroemer G. Science 2014; 345: 1250256.

4. Galluzzi L et al. Nat Rev Drug Discov 2013; 12: 829-846.

5. Jiang $L$ et al. Nature 2015; 520: 57-62.

6. Li T et al. Cell 2012; 149: 1269-1283.

7. Dixon SJ et al. Cell 2012; 149: 1060-1072.

8. Yang WS, Stockwell BR. Chem Biol 2008; 15: 234-245.

9. Dixon SJ et al. Elife 2014; 3: e02523.

10. Galluzzi L et al. 2015 Cell Death Differ 2015; 22: 58-73.

11. Galluzzi $L$ et al. Cell Death Differ 2012; 19: 107-120.

12. Galluzzi $L$ et al. EMBO J 2015; 34: 856-880.

13. Vandenabeele P et al. Nat Rev Mol Cell Biol 2010; 11: 700-714.

14. Vanden Berghe T et al. Nat Rev Mol Cell Biol 2014; 15: 135-147.

15. Bonora M W et al. Oncogene 2015; 34: 1475-1486. 